\title{
EFFETS PHYSIOLOGIQUES DES EXPLOSIONS SUR LES ANIMAUX AQUATIQUES
}

\author{
par G. de la FOUCHARDIËRE \\ Ingénieur principal des Eaux et Forêts.
}

Ayant eu récemment l'occasion d'employer sous l'eau des quantités importantes d'explosifs, pour la destruction d'une épave qui, en baie de Saint-Brieuc, gênait la navigation, j'ai pu me livrer, à ce sujet, à un certain nombre d'observations sur les effets physiologiques des explosions sous-marines, effets qui, jusqu'à présent, ne semblent pas avoir beaucoup préoccupé les chercheurs, puisqu'à ma connaissance aucun texte ne les relate et qu'il n'existe comme documentation à ce sujet qu'une trop courte séquence dans l'admirable film de Cousteau "Le Monde du silence ".

Au cas présent, des charges unitaires de dynamite-gomme de 10 kilos ont été mises à feu à une profondeur moyenne d'une dizaine de mètres, sur l'épave d'un navire en bois de 35 mètres de longueur qui, dans une mer particulièrement poissonneuse était, comme toutes les épaves d'ailleurs, le lieu de rassemblement d'un nombre considérable de poissons.

Ces explosions ont eu lieu tard en saison (Octobre) et malheureusement à cette époque le nombre d'espèces présentes en pleine eau [Maquereaux et Saurels (Trachurus)] était assez faible.

Dans le remous provoqué par l'épave au milieu du courant de marée variant toutes les six heures, tant en intensité qu'en direction et qui, par moment, atteignait plusieurs mètres à la seconde, évoluaient des gadidés : Lieus (Pollachius pollachius) représentés par quelques individus de forte taille et surtout Tacauds (Gadus luscus) de 10 à 200 grammes rassemblés en essaims innombrables.

Sur le fond, et notamment dans les anfractuosités de l'épave, une quantité importante de Congres qui occupaient tous les logements possibles et étaient représentés par des individus allant de 1 à 15 kilos ; quelques Vieilles et également de nombreuses Blennies (Blennius gattorynchus) espèce très voisine de l'espèce dulcaquicole broutant les goémons et mangeant les mollusques.Ces poissons ont été observés lors des plongées précédant la mise à feu. 
Aussitôt après chaque explosion, on vit remonter en surface une quantité de poissons difficile à évaluer, mais qui devait représenter 100 à 200 kilos au total et qui furent en partie capturés.

Dès que le nuage de vase soulevé par l'explosion fut un peu dissipé par le courant, une plongée permit de constater qu'un nombre encore plus considérable d'individus gisaient sur le fond, morts ou mourants et qu'il n'y avait pas toujours de différence spécifique entre certaines espèces flottant et d'autres retombant au fond, ce qui à priori nous a semblé tout à fait curieux. Un poisson, en effet, hors le cas très rare où il flotte inerte à la surface, cas notamment du Mole, est entre deux eaux ou repose sur le fond; les Tacauds et les Lieus, notamment, ne sont jamais en contact avec le fond et, sans l'aide de leurs nageoires, restent parfois parfaitement immobiles à une profondeur donnée par le jeu de leur vessie natatoire, dont consciemment ou non ils font varier le volume pour augmenter ou diminuer leur densité selon la profondeur.

Il est à remarquer que d'autres poissons, comme les Scombres, qui sont dépourvus de vessie natatoire, ne se maintiennent que par un mouvement incessant de leurs nageoires, ayant une densité légèrement supérieure à celle de l'eau. Il était donc normal de trouver sur le fond, et seulement là, les Maquereaux et quelques Saurels victimes de l'explosion, et que tous les Lieus retrouvés morts soient remontés le ventre en l'air.

Par contre, pour les Tacauds, la proportion est à peu près moitiémoitié. Une explication est possible : lorsqu'un poisson dont la densité, à une profondeur donnée, différe très peu de celle de l'eau qui l'environne, est tué, l'état d'équilibre dans lequel il se trouve est rompu et plus il remontera, plus sa vessie natatoire se dilatant, sa densité diminue et sa remontée devient rapide (comme la " remontée en ballon "redoutée des scaphandriers).

Un exemple particulièrement spectaculaire est donné lorsqu'un chalut dans lequel se trouvent plusieurs tonnes de Morues est hâlé vers la surface à la fin des traits : les poissons se dilatant au fur et à mesure de cette remontée, finissent par faire flotter l'engin lui-même, que l'on voit brusquement apparaître en surface la poche la première, alors que les treuils ont encore parfois plus de 100 mètres de funes à rentrer.

L'on comprend aisément le mécanisme qui fait arriver à l'air libre les poissons se trouvant au-dessus ou au niveau de la charge, notamment les Lieus qui sont toujours à mi-chemin entre le fond et la surface. Par contre, si un poisson en état d'équilibre est projeté vers des zones plus profondes, sa densité augmente progressivement au fur et à mesure qu'il s'enfonce et on le retrouve normalement sur le fond.

Il est donc logique, selon ce principe, de retrouver après l'explosion sur un bateau, à peu près $50 \%$ des Tacauds de chaque côté, ce qui correspond environ à leur répartition dans l'eau par rapport au niveau de la charge.

Les poissons du type démersal ont toujours une densité supérieure a celle de l'eau et reposent sur le fond dont ils ne peuvent se décoller que 
par un effort musculaire. C'est le cas des Blennies dont aucun cadavre ne fut retrouvé en surface alors que le fond en était par endroits littéralement couvert.

Or, en fait les explications données précédemment ne sont plus du tout satisfaisantes, lorsqu'on regarde le cas du Congre.

C'est un poisson démersal qui est toujours dans une cavité et dont la densité est très supérieure à celle de l'eau, même lorsqu'il a subi une décompression importante. C'est ainsi, par exemple, que l'on constate qu'un de ces animaux pris à la ligne dans 20 ou 25 mètres d'eau et amené très brutalement en surface, garde néanmoins une densité très importante malgré la décompression, puisque, si la ligne se décroche au moment de l'embarquer, on voit le poisson, rigoureusement immobile, repartir vers les profondeurs au grand dam du pêcheur, alors qu'un Labridé dans les mêmes conditions n'arrive pas, malgré tous ses efforts, à quitter la surface et continuera à flotter, le ventre distendu par l'expansion des gaz internes.

Or, après une explosion sous-marine, la proportion de Congres remontant à la surface est à peu près la même que pour des espèces de pleine eau. Il y a là une anomalie difficile à expliquer.

Quelles sont les lésions subies par les poissons lors d'une explosion sous-marine?

Une explosion produit une onde de choc caractérisée par une compression considérable suivie d'une décompression immédiate. En dehors de la zone extrêmement proche de la charge $(3 \mathrm{~m}$. par exemple pour $10 \mathrm{~kg}$. de dynamite-gomme) il n'y a pas de déplacement visible des objets et à fortiori du poisson. Par contre, l'effet de choc doit se faire sentir fort loin, puisqu'on le perçoit très bien si on laisse tremper la main dans l'eau alors même que l'on s'est éloigné, pour des raisons de sécurité, à 150 mètres du lieu de l'explosion; à cette distance d'ailleurs l'on se rend très bien compte de la vitesse de propagation du choc dans l'eau et du son dans l'air avec quelques dizièmes de secondes de retard.

Dans la zone A très rapprochée $(1 \mathrm{~m}$.) rien ne subsiste; c'est une zone d' "écrabouillement " où tout est déchiqueté, qu'il s'agisse d'une pièce de bois ou d'un organisme vivant. Dans une zone $B$, plus éloignée (3 à $4 \mathrm{~m}$.), il y a déplacement, mais sans effet de rupture. Les pièces de bois sont disjointes, le chevillage est arraché ; il y a également décapage de toute l'incrustation benthique qui, au cas présent, atteignait plus d'un centimètre d'épaisseur (Coraux, Gorgones, Cirripèdes, Mollusques, etc...) : c'est la zone mortelle pour le poisson.

Très rapidement on voit les effets s'atténuer et il est surprenant de voir que, malgré la violence de l'explosion et l'abondance de poissons trouvés morts, il en reste une quantité tout aussi considérable qui, apparamment, n'ont subi aucun mal, aucun cadavre n'ayant été trouvé à plus de 10 mètres de la charge (zone $\mathrm{C}$ ).

Des autopsies de poissons ont été faites sur place et ont donné les résultats suivants :

- Dans la zone A, il ne reste que des lambeaux méconnaissables. 
Dès qu'on s'en éloigne, l'on peut trouver des poissons littéralement disloqués présentant notamment de très nombreuses fractures de la colonne vertébrale (les autopsies sur les poissons sont extrêmement faciles car toute lésion traumatique se manifeste par un épanchement sanguin tranchant nettement sur la couleur blanche de la chair). Les fractures de colonne vertébrale ne s'étendent pas au-delà de la zone B et beaucoup de poissons en sont indemnes : il semble que leur mort soit due à une autre cause.

Je n'ai pas trouvé un seul cas de rupture de la vessie natatoire, ce qui est normal étant donné l'élasticité même de cet organe et ce qui est contraire à l'opinion généralement admise selon laquelle les poissons sont tués par éclatement de la vessie, dont le gaz répandu dans la cavité générale les fait ultérieurement flotter.

Enfin, l'immense majorité des poissons recueillis tant en surface que sur le fond, ne présente aucune lésion apparente. Ils ont donc pu être pratiquement assommés et seule une autopsie très minutieuse pourrait déceler des lésions craniennes.

Cette raison doit être prépondérante car si beaucoup de poissons sont effectivement morts, l'on en voit néanmoins un certain nombre qui, étant " $k$. o. pour le compte " dirait-on en parlant d'un boxeur, s'animent et au bout de quelque temps reprennent une vie tout à fait normale; l'on voit sur le fond des Tacauds couchés sur le côté qui, peu à peu, recommencent à s'agiter, les premiers mouvements étant ceux des opercules qui indiquent que la respiration normale est rétablie, puis des nageoires paires et enfin le poisson reprend sa position verticale et peu à peu son comportement habituel.

Le cas est encore plus marqué pour des espèces dont la vitalité est bien connue comme le Congre : capturés flottant inertes en surface et embarqués, certains d'eutre eux, au bout de quelques dizaines de minutes, avaient repris vie et se montraient même agressifs. Quelquesuns remis à l'eau ont immédiatement montré un comportement absolument normal.

L'effet de l'explosion avait eu pour eux exactement les mêmes conséquences que le coup de maillet sur le crâne, indispensable pour pouvoìr décrocher de la ligne ces sujets encombrants dont la machoire est redoutable.

On peut d'ailleurs y assimiler également le procédé de braconnage qui consiste à frapper violemment avec une masse les rochers se trouvant dans le lit de la rivière, provoquant une onde de choc s'étendant à l'eau et assommant les Truites pouvant se trouver à proximité immédiate.

En ce qui concerne les invertébrés, les effets immédiats de l'explosion sont beaucoup moins sensibles.

En dehors de la zone $A$, où tout est écrasé, l'on constate dans la zone B un décapage des bois qui apparaissent parfaitement propres par décollement de l'incrustation benthique qui comportait une infinité d'organismes vivants, animaux ou végétaux dont l'énumération complète serait fastidieuse. Mais ces animaux, après l'explosion, semblaient vivre de façon tout à fait normale. 
Parmi les Mollusques, les Bivalves (Moules, Hanards) n'avaient absolument pas souffert; les Gastéropodes, qui étaient représentés par diverses espèces de Littorines, des Nasses et des Ormeaux, étaient toujours parfaitement vivants.

Les Céphalopodes, qui représentent des organismes beaucoup plus évolués, semblent pourtant être plus sensibles, puisque j'ai retrouvé des Pieuvres (Octopus) et des Seiches tuées par l'explosion mais seulement dans un faible rayon autour de l'emplacement des charges.

Les Crustacés ont paru également totalement insensibles, qu'il s'agisse des Crustacés supérieurs (Décapodes), puisque Crevettes (Leander) aussi bien que Crabes (Carcinus et Portunus) continuent à évoluer et qu'aucun cadavre n'en a été retrouvé, ou des Crustacés inférieurs, tels que les Isopodes représentés notamment par des Limnorias incrustant les bois ou les Cirripèdes qui forment d'énormes colonies. Il en est exactement de même pour les Echinodermes particulièrement bien représentés par de nombreuses espèces d'Ophiures, d'Oursins, d'Astéries ou de Comatules. Aucun effet visible sur les animaux inférieurs tels que Tuniciers, Bryozoaires, Gorgones, etc.

Une plongée effectuée 20 jours plus tard nous a fait voir des conséquences lointaines absolument inattendues.

A peine le fond était-il atteint que l'on était surpris de voir que l'épave qui grouillait de vie, même immédiatement après les explosions était devenue un désert. Il ne restait plus que quelques dizaines de Tacauds évoluant d'ailleurs assez loin de l'épave et, même en explorant soigneusement les amoncellements de bois qui auraient pu leur offrir des retraites, on n'a pu voir aucun Congre.

Ce qui frappe également, c'est de voir que beaucoup des organismes qui n'avaient pas souffert au moment même de la déflagration, étaient maintenant privés de vie.

Si les Moules restaient bien vivantes, les Hanards étaient morts, ainsi que les Crustacés Cirripèdes ou Isopodes et la plupart des organismes fixés tels que Éponges, Gorgones ou Bryozoaires (dont il existait de magnifiques exemplaires d'Escharia foliacea) qui apparaissaient réduits à leurs supports blanchis au lieu d'offrir les merveilleuses colorations habituelles.

Parmi les Gastéropodes, les Littorines semblaient avoir diminué légèrement d'importance. Par contre, il $\mathrm{y}$ avait une abondance extraordinaire de Nasses, ce qui est normal puisque ce Gastéropode est un charognard : les restes putréfiés de poissons qui jonchaient le sol en étaient littéralement couverts.

Parmi les Crustacés, les Étrilles (Porlunus) avaient disparu; par contre, les Crabes verts étaient particulièrement nombreux. Les Échinodermes étaient réduits aux seules Ophiures.

Sans s'étendre longuement sur le cas particulier de chaque espèce, la constatation immédiate était celle-ci : l'explosion n'avait pas provoqué la mort mais une pollution consécutive à la putréfaction de tous les poissons tués (ou à la mobilisation de la vase) avait empoisonné les orga- 
nismes fixés et chassait ceux qui, libres, pouvaient fuir une eau chargée de principes toxiques.

C'est ainsi que l'on peut expliquer des effets différents sur des organismes très voisins l'un de l'autre. En ce qui concerne par exemple le Hanard et la Moule qui ont, l'un comme l'autre, fort bien résisté à l'explosion, mais alors que le Hanard qui est toujours un Mollusque d'eaux propres avait péri ensuite, les Moules qui ne craignent pas la pollution puisque les plus belles se trouvent à la sortie des égouts, étaient restées bien vivantes. La même chose en ce qui concerne les Crabes : l'Étrille ne se trouve que sur des fonds de roche ou herbiers dans des eaux claires, alors que le Crabe vert est l'habitant normal des fonds de port vaseux où il nettoie les carcasses de poisson en décomposition.

Cet effet secondaire de l'explosion peut avoir une très grande importance car pour avoir été si violent en mer, où la dilution est infinie, où l'eau est constamment brassée et oxygénée par l'action du vent et où de plus un courant très sensible renouvelle l'eau de manière incessante, l'on voit ce qui doit se produire dans les eaux douces où la pollution peut avoir des conséquences beaucoup plus graves et beaucoup plus durables.

En conclusion, l'expérience qui a pu être faite, et qui malheureusement est unique, a apporté les enseignements suivants :

10 Malgré les grosses quantités d'explosifs utilisées (par paquets de 100 cartouches à la fois) les effets de l'explosion sont très vite amortis dans l'espace et l'effet ne se fait sentir que dans un rayon de quelques mètres.

La quantité de poisson remontant à la surface et qui représente le résultat tangible dans le cas d'un braconnage, est minime par rapport à celle qui reste au fond de l'eau.

$2^{\circ}$ Cette remontée est d'ailleurs très variable suivant les espèces, certaines coulant toutes, d'autres flottant toutes, la plupart se divisant de manière variable puisqu'un certain nombre d'individus remontent en surface, alors que d'autres restent dans les profondeurs.

$3^{\circ}$ Les invertébrés sont très peu sensibles aux effets de l'explosion.

$4^{\circ}$ A la suite de la décomposition du poisson tué et restant sur le fond, et peut-être pour d'autres raisons qu'il serait intéressant d'étudier (remontée de vase, mort du Plancton invisible à l'œil nu, etc.) il se produit une pollution intense amenant la destruction de la plupart des organismes fixés, sauf lorsqu'il s'agit d'espèces pouvant vivre dans des eaux chargées de matières organiques.

La région où l'explosion eut lieu reste donc, à l'exception des animaux d'eaux sales, un désert pendant une assez longue période, même lorsqu'il s'agit d'eaux profondes brassées par la houle et renouvelées par les courants, ce qui minimise cette pollution.

Une plongée effectuée 3 mois après l'explosion a permis de confirmer ces conclusions : la vie était redevenue normale, et seuls le décapage des bois et la présence de coquilles vides, squelettes et restes blanchis 
sur lesquels une nouvelle incrustation commençait déjà, attestaient le cataclysme biologique dont l'épave avait été le théâtre.

Il est peut-être difficile d'extrapoler aux eaux douces une expérience faite en mer dans des conditions tout à fait différentes.

Cependant, on pourrait logiquement en déduire que bien que la vie soit beaucoup moins intense dans les eaux douces, puisque la densité de poissons n'y est pas comparable et que, d'autre part, il n'existe pas une incrustation benthique comme en mer, les effets de la pollution consécutive à l'explosion seraient beaucoup plus considérables dans les eaux dormantes peu profondes étant donné la faible dilution et le fait que l'eau ne se renouvelle pas.

L'on pourrait probablement constater une pollution descendante plus ou moins grave dont les effets ne semblent pas avoir été signalés; lorsqu'il y a délit de braconnage à l'explosif, les constatations sont toujours faites dans un très court délai après le délit et il semblerait très intéressant de les continuer pendant plusieurs semaines, notamment en aval, pour voir si, en dehors des poissons tués par l'explosion ellemême, il n'y a pas d'autres dégâts qui risquent d'être plus durables et beaucoup plus importants. 